This is the accepted manuscript of the article,

which has been published in Acs biomaterials

science and engineering. 2019, vol 5(2), 1134-1140.

https://doi.org/10.1016/j.ipm.2019.102080

\title{
Bioresorbable conductive wire with minimal metal
}

\section{content}

Aleksi Palmroth ${ }^{1, *}$, Timo Salpavaara ${ }^{1}$, Inari Lyyra ${ }^{1}$, Mart Kroon ${ }^{1}$, Jukka Lekkala ${ }^{1}$, Minna

Kellomäki ${ }^{1,2}$

${ }^{1}$ BioMediTech Institute and Faculty of Biomedical Sciences and Engineering, Tampere

University of Technology, Tampere 33720, Finland.

${ }^{2}$ BioMediTech Institute and Faculty of Medicine and Life Sciences, University of Tampere, Tampere 33104, Finland.

*Corresponding author: aleksi.hanninen@tut.fi

\section{KEYWORDS}

Transient electronics, bioresorbable, conductive wire, extrusion, RF coil

\section{ABSTRACT}

The emergence of transient electronics has created the need for bioresorbable conductive wires for signal and energy transfer. We present a fully bioresorbable wire design where the conductivity is provided by only a few micrometers thick electron-beam evaporated magnesium layer on the surface of a polymer fiber. The structure is electrically insulated with an extrusion coated polymer sheath, which simultaneously serves as a water barrier for the dissolvable magnesium conductor. 
The resistance of the wires was approximately $1 \Omega \mathrm{cm}^{-1}$ and their functional lifetime in buffer solution was more than one week. These properties could be modified by using different conductor materials and film thicknesses. Furthermore, the flexibility of the wires enabled the fabrication of planar radio frequency coils, which were wirelessly measured. Such coils have the potential to be used as wireless sensors. The wire design provides a basis for bioresorbable wires in applications where only a minimal amount of metal is desired for example to avoid toxicity.

\section{INTRODUCTION}

Implantable conductive wires and cables provide the means for accessing difficult sensing and stimulation locations inside the body ${ }^{1}$ for example in cases where the working distance for wireless devices is too long. In addition, conductors are needed in fabricating radio-frequency (RF) coils, which can be used in resonating sensor structures ${ }^{2}$ or to transmit power wirelessly to implantable medical devices ${ }^{3}$. Implantable wires typically comprise metal alloy based core conductors and an insulating layer protecting the conductors from biological fluids ${ }^{1}$. In long-term applications, their robustness and durability are crucial. On the other hand, the emergence of bioresorbable implantable devices ${ }^{4}$ for monitoring or treating acute health conditions has created a need for conductive wires that disappear from the body after functioning for a defined time period.

In conductive wire and cable applications, biodegradable materials have often been used only in the sheath. Previous studies include for example an extrusion coated poly(L-lactide) sheath on a copper wire ${ }^{5}$ and cellulose sheaths around carbon nanotube cores $^{6}$. On the contrary, preclinical studies on biodegradable implantable sensors have been conducted using biodegradable transcutaneous molybdenum based wires for signal transfer ${ }^{7,8}$. 
An implantable conductive wire with only minimal bioresorbable metal content might be useful in applications where excessive amounts of metals could cause problems. For example, the corrosion of magnesium produces hydrogen gas, which might lead to complications in vivo ${ }^{9}$. Zinc on the other hand is known to be neurotoxic and hinder bone development at immoderate quantities ${ }^{10}$. Furthermore, some bioresorbable metals like iron dissolve very slowly and are even noticed to cause similar problems that are observed with permanent implants ${ }^{11}$. These issues might be solved by depositing metallic thin films on bioresorbable polymer fibers to achieve conductivity. Depending on the application and the conductivity of the used metal, the metal thickness could be for example from a few hundred nanometers to a few micrometers. The resulting wire can be extrusion coated without destroying the conductivity, if the glass transition temperature of the core fiber is higher than the melting temperature of the coating material. The mechanical properties of such conductive wires are largely defined by the polymer materials, but at the same time constrained by the integrity of the conductive metal layer.

In this study, prototype wires were fabricated using bioresorbable poly(desamino tyrosyltyrosine ethyl ester carbonate), or poly(DTE carbonate) as the core fiber material owing to its relatively high glass transition temperature. After depositing magnesium onto the core fiber, it was extrusion coated using bioresorbable poly(caprolactone) (PCL). The obtained wires were characterized by demonstrating their conductivity, flexibility and lifetime in aqueous conditions. Furthermore, their application as wirelessly readable RF coils was shown. Such coils could be used for example as sensors to detect changes in their environment.

\section{EXPERIMENTAL SECTION}

\section{Wire fabrication}


Poly(desamino tyrosyl-tyrosine ethyl ester carbonate) (poly(DTE carbonate); Integra LifeSciences, New Jersey, USA) with a measured inherent viscosity (i.v.) of $0.89 \mathrm{dl} / \mathrm{g}$ was dried in vacuum for four days in room temperature before processing. A flat poly(DTE carbonate) monofilament fiber was extruded under nitrogen atmosphere using Gimac microextruder (Gimac, Gastronno, Italy) with a screw diameter of $12 \mathrm{~mm}$. The shape and dimensions of the extrusion die are presented in Figure S1. The flat shape was chosen to prevent any unintentional twisting of the fibers during the subsequent metal deposition process. The fiber was collected with a conveyer at a speed of $4 \mathrm{~m} / \mathrm{min}$. The thickness and width of the poly(DTE carbonate) core fiber and the complete conductive wire were measured from different points $(\mathrm{n}=50)$ using a digital caliper.

Continuous poly(DTE carbonate) fibers were cleaned using isopropanol dampened clean room wipes and then coiled around a holder plate using Kapton tape for attachment. Magnesium thin films with a thickness of $7.5 \mu \mathrm{m}$ were e-beam evaporated onto the fibers. This was close to the maximum Mg thickness that could be conveniently evaporated during one process using our system. The e-beam evaporation rate was $2.0 \AA / \mathrm{s}$ with a vacuum level in the order of $10^{-6}$ torr. After evaporation, the Kapton tapes were removed, resulting in poly(DTE carbonate) fibers with various lengths $(9-14 \mathrm{~cm})$ of continuous magnesium coating limited by the tape. The fibers with a magnesium layer were extrusion coated with medical grade poly(caprolactone) (PCL; Corbion Purac, The Netherlands) having an i.v. of $1.18 \mathrm{dl} / \mathrm{g}$ as stated by the manufacturer. The extrusion was performed with the Gimac microextruder under nitrogen atmosphere using a crosshead die with an output diameter of $2 \mathrm{~mm}$. The resulting wire was collected with a conveyer at a speed of $3 \mathrm{~m} / \mathrm{min}$.

\section{Materials characterization}


The thermal transitions of the poly(DTE carbonate) and PCL raw materials were determined using a differential scanning calorimeter (DSC Q1000; TA Instruments, USA). The data was analyzed from the second heating using TA Universal Analysis software.

The inherent viscosity measurements of the extruded poly(DTE carbonate) core fiber and the respective raw material were performed to evaluate the degradation of the material during processing. The analysis was done in ambient conditions using an automated Ubbelohde viscometer (LAUDA, Lauda-Königshofen, Germany). Samples of $20 \pm 0.6 \mathrm{mg}$ were weighed and dissolved in $20 \mathrm{ml}$ of chloroform overnight before the measurement. Two parallel samples for both the raw material and the fiber were measured.

Compression molded poly(caprolactone) sheets $(\mathrm{n}=5)$ with a size of $10 \times 50 \times 0.4 \mathrm{~mm}^{3}$ were used to study the water uptake properties of the PCL extrusion coating material. The samples were dried in a vacuum chamber at room temperature for one week, after which their mass was weighed using an analytical scale. The samples were then immersed in Sörensen buffer solution (Table S1), which was prepared according to the "ISO 15814:1999(E): Implants for surgery - Copolymers and blends based on polylactide - In vitro degradation testing" standard. The immersed samples were stored at $+37^{\circ} \mathrm{C}$ and weighed at pre-determined time points by gently wiping the excessive liquid from the samples with tissue paper and immediately weighing them. The wet weights were compared to the initial dry weight using equation 1 :

$$
\text { Water uptake }(\%)=\left(\frac{\text { Wet weight-Dryweight }}{\text { Dryweight }}\right) \times 100 \% \text {. }
$$


The possible error caused by the mass loss of PCL during hydrolysis was considered negligible, as virtually no molecular weight changes and an average mass loss of only $0.12 \%$ per month have been reported for the first 6 months of PCL hydrolysis ${ }^{12}$.

The tensile properties of both the plain core fibers and the PCL coated fibers $(n=10)$ were studied using Instron 4411 materials testing equipment (Instron Ltd., England) with a $500 \mathrm{~N}$ load cell. The distance between pneumatic grips was $50 \mathrm{~mm}$ and the crosshead speed was $10 \mathrm{~mm} / \mathrm{min}$. The cross sectional areas of the fibers were approximated using a rectangular shape as a basis. Tensile strength, elongation at break and Young's modulus were calculated from the measured data.

Scanning Electron Microscopy

The scanning electron microscopy (SEM) images were taken from the cross section of the conductive wire. The wire was first immersed in methanol to fill the material (meso)pores and thus promote brittleness during cutting ${ }^{13}$. After the methanol treatment the wire was freeze fractured in liquid nitrogen, coated with a thin layer of chromium and imaged using a field emission SEM (JSM-7610F; JEOL Ltd., Japan). The imaged sample originated from an earlier fabrication batch compared to the samples used in other tests.

\section{Resistance measurements}

After evaporating magnesium onto the fibers, their resistance was measured using a digital multimeter (AxioMET AX-572) by gently pressing the probes onto the Mg layer. The length of the magnesium coatings, defined by the tapes in the evaporation phase, were measured using a ruler. The resistance per centimeter was calculated for each conductive fiber $(n=21)$. The measurements and calculations were repeated after extrusion coating in order to evaluate the effect 
of coating process to the wire resistance. Excessive PCL coating was first melted and skimmed off the tip of the wires, after which the multimeter probes were pressed through the residual PCL film. A paired Student's t-test was applied for the statistical analysis, where $\mathrm{p}<0.05$ was considered significant.

After the dry resistance measurements, the tips of the wires were treated with two layers of conductive silver epoxy adhesive (8331-14G; MG Chemicals, Canada) to ensure a measurement point for the water immersion tests. The bioresorbable wires $(\mathrm{n}=5)$ were then attached to $50 \mathrm{ml}$ falcon tubes, leaving the Ag epoxy coated wire tips in air and the rest of the wire immersed in prewarmed Sörensen buffer solution. The samples were stored in an incubator at $+37^{\circ} \mathrm{C}$ and their resistances were measured with a multimeter using hook clip probes.

\section{Bending and resonator coils}

Flexibility is essential for implantable wires. However, bending should not compromise their conductivity. This aspect was tested by wounding the wires around a glass rod with a diameter of $7 \mathrm{~mm}$ as shown in Figure 1a. The resistances of the wires $(\mathrm{n}=5)$ were measured with a multimeter before and after the bending experiment. 


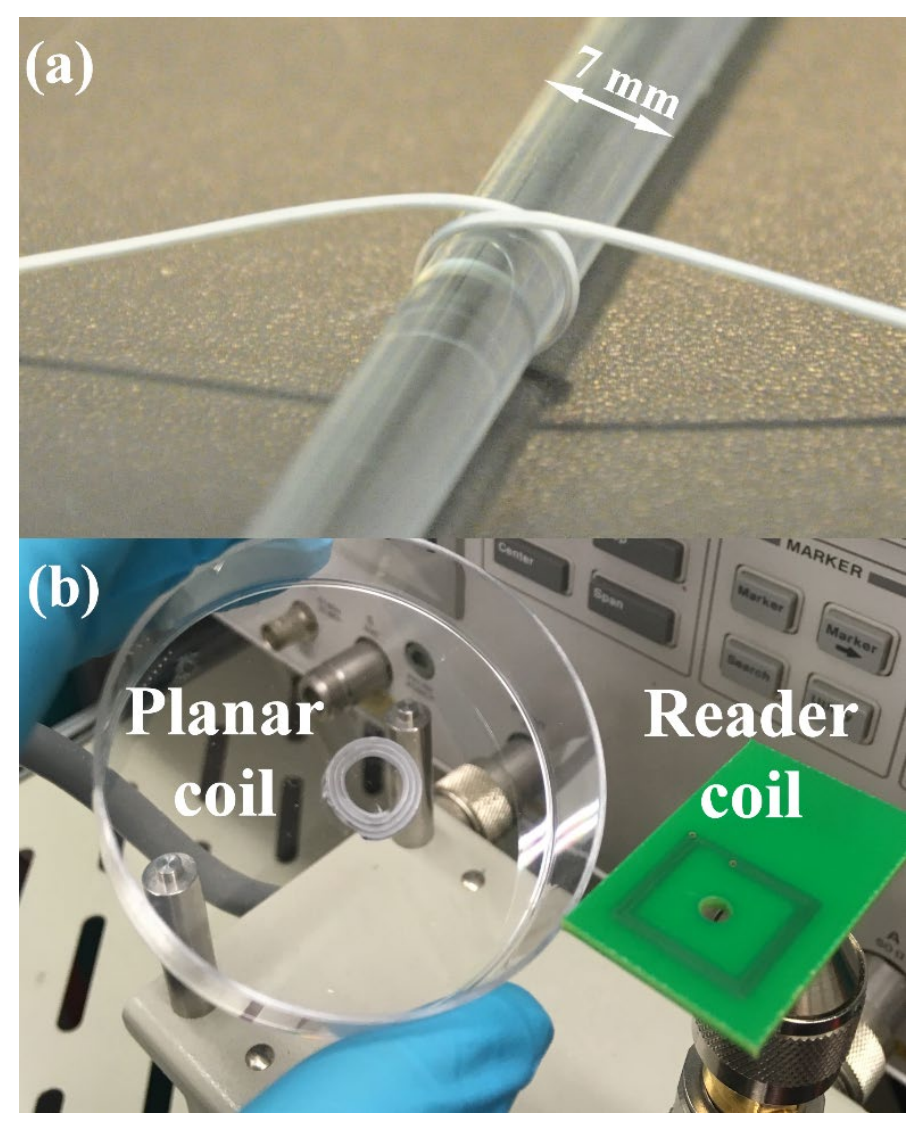

Figure 1. The wire flexibility demonstrations showing (a) a wire coiled around a glass rod with a $7 \mathrm{~mm}$ diameter and (b) a planar coil with an inner diameter of $7 \mathrm{~mm}$ and the external reader coil used for the wireless resonance measurements.

One of the simplest functional structures that can be fabricated from conductive insulated wires is a coil. These coils are sensitive to their environment ${ }^{14}$ and can be used for example as sensors. The self-resonance frequencies of the coils can be wirelessly estimated by measuring a reader coil that is inductively coupled with the sensor coil.

Three bioresorbable planar coils with three turns were fabricated from the conductive wires. First, the wires were coiled around a 3D printed template with a diameter of $7 \mathrm{~mm}$. Then, a soldering iron was used to melt the PCL coating, which acted as an adhesive after cooling. In addition, three non-degradable reference samples were fabricated from insulated copper wire (Flexi-E 0.10, 
Stäubli Group, Switzerland) using the same template and super glue as an adhesive. This commercial copper-based cable consisted of a non-degradable insulating polyvinyl chloride (PVC) sheath and a stranded $\mathrm{Cu}$ core with a conductor diameter of $0.40 \mathrm{~mm}$ and a total diameter of 1.0 $\mathrm{mm}$.

The resonance frequencies of all the bioresorbable and non-degradable coils were first wirelessly measured through a plastic petri dish using an external two-turn reader coil (Figure 1b). An impedance analyzer (Agilent 4396B; $100 \mathrm{kHz}-1.8 \mathrm{GHz}$ ) was used to measure the real part of the impedance of the reader coil from $650 \mathrm{MHz}$ to $1 \mathrm{GHz}$. Thereafter, the reading distances of one bioresorbable coil and one non-degradable coil were tested. The coils with the highest resonance frequencies were chosen for this test. The reading distance was increased in a stepwise manner by adding $1 \mathrm{~mm}$ thick microscopy slides between the coil and the reader. The coils were measured after each $1 \mathrm{~mm}$ increment.

\section{RESULTS AND DISCUSSION}

\section{Wire Structure and Processing}

Fabrication of the conductive wire started by extruding poly(DTE carbonate) to form the core fiber. Based on an SEM image of the wire cross section (Figure 2), the poly(DTE carbonate) core took accurately the shape of the extruder die. The core located in the lower portion of the PCL sheath with an approximately $50 \mu \mathrm{m}$ thick coating layer in the thinnest region. As measured using the digital caliper, the average $(\mathrm{n}=50)$ width of the core fibers was $0.79 \pm 0.03 \mathrm{~mm}$ and thickness $0.29 \pm 0.02 \mathrm{~mm}$, whereas the same dimensions for the complete wire including the extrusioncoated sheath were $1.05 \pm 0.05 \mathrm{~mm}$ and $0.73 \pm 0.09 \mathrm{~mm}$, respectively. The dimensions in the SEM image differ from these measurements, showing a core fiber width of approximately $0.9 \mathrm{~mm}$. The 
SEM sample was prepared from a preliminary fibers batch, where the fibers were collected at a lower conveyer speed. The fibers from the preliminary test batch showed similar behavior with the reported fibers. The dimensions of both the core and the sheath could be tuned by modifying the extruder dies or collecting the fibers at a different rate. For example, Bourke et al. have extruded poly(DTE carbonate) fibers with a diameter of $61 \mu \mathrm{m}^{15}$.

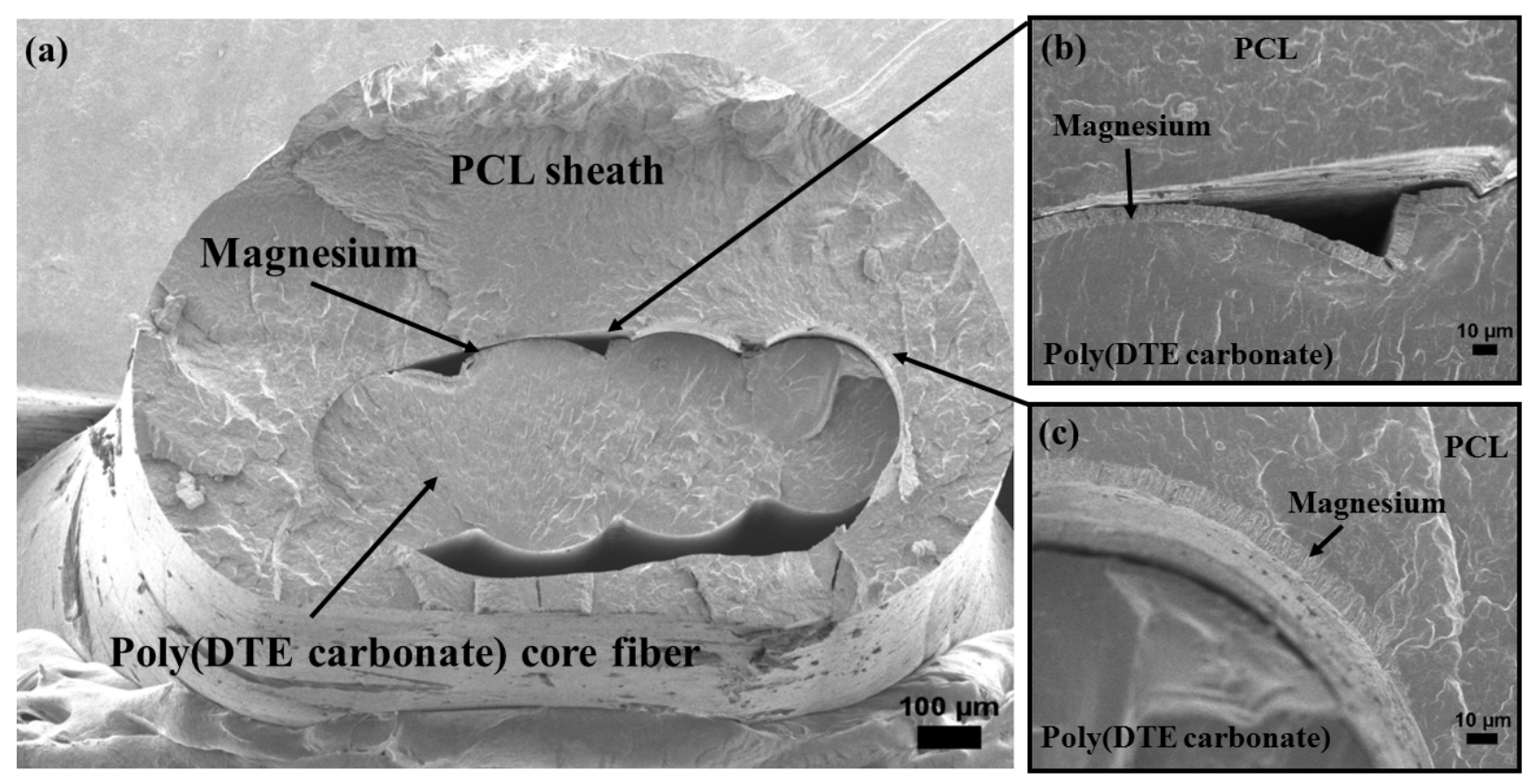

Figure 2. Cross section of the conductive wire showing (a) the whole cross section, (b) magnesium film on top of the core fiber and (c) magnesium attached to the PCL sheath.

The e-beam evaporated magnesium layer appeared to have a relatively uniform thickness of 7-8 $\mu \mathrm{m}$ also in the grooves of the core fiber (Figure $2 \mathrm{~b}$ ). Interestingly, some parts of the magnesium had a greater adhesion on PCL than on the poly(DTE carbonate) core (Figure 2c), causing partial detachment of the Mg layer from the core fiber. We estimate that the detached Mg layers have been surrounded by molten PCL in the extrusion coating phase and have possibly detached during the cooling phase of PCL or even later when cutting the SEM sample. However, the PCL sheath was not fully in contact with the core fiber and specifically did not reach the grooves of the core 
fiber, which should remain conductive even if larger flakes would be detached. However, this issue might be improved by using adhesion promotion methods such as plasma treatment before metal evaporation.

Based on the shape of the sheath, we conclude that the lack of contact at some locations existed already before cutting the wire for the SEM imaging. The lengths of the conductive wires were limited by the Kapton tapes that were used to attach the fibers to the holder in the evaporation phase. Reeling the core fiber onto a spool that rotates in the evaporation chamber would enable fabricating wires with meters of length. Longer continuous wires could be made for example by using a roll-to-roll sputtering deposition ${ }^{16}$ process.

\section{Materials characterization}

As an amorphous polymer ${ }^{17}$, poly(DTE carbonate) does not have a melting temperature. Its glass transition temperature $\left(\mathrm{T}_{\mathrm{g}}=99{ }^{\circ} \mathrm{C}\right)$ is higher than the melting temperature of $\mathrm{PCL}$ $\left(\mathrm{T}_{\mathrm{m}}=57^{\circ} \mathrm{C}\right)$, enabling the extrusion coating process without damaging the core fiber by excessive heating. Within this constraint $\left(\mathrm{T}_{\mathrm{g} \text {, core }}>\mathrm{T}_{\mathrm{m}}\right.$, sheath $)$, the material choices in the wire can be tuned if for example faster degradation is preferred.

The tensile properties of the core fiber were examined to assess the extrusion process. The tensile properties of the extrusion products are illustrated in Table 1. The tensile strength of our fibers (56 $\pm 3 \mathrm{MPa}$ ) was slightly higher than the $44 \pm 11 \mathrm{MPa}$ of the first-generation poly(DTE carbonate)

fibers of Bourke et al. ${ }^{15}$ However, the Young's Modulus of our fibers (1.9 GPa) was lower compared with their second-generation fibers $(3.1 \mathrm{GPa})$ with a diameter of $79 \mu \mathrm{m}$. Both the Young's Modulus and tensile strength were lower in the PCL coated fiber. 
Table 1. Properties of the plain and the extrusion coated core fibers as averages \pm standard deviations.

\begin{tabular}{|l|l|l|l|}
\hline & $\begin{array}{l}\text { Young's } \\
\text { Modulus (GPa) }\end{array}$ & $\begin{array}{l}\text { Tensile strength } \\
\text { (MPa) }\end{array}$ & $\begin{array}{l}\text { Elongation at } \\
\text { break (\%) }\end{array}$ \\
\hline Poly(DTE carbonate) core fiber & $1.9 \pm 0.1$ & $56 \pm 3$ & $4.2 \pm 0.2$ \\
\hline PCL coated core fiber & $0.9 \pm 0.1$ & $25 \pm 3$ & $4.0 \pm 0.4$ \\
\hline
\end{tabular}

The tensile properties of the core fiber could be significantly modified by adding a further drawing step. Orientation of semicrystalline polymer fibers typically increases the Young's Modulus and tensile strength parallel to the drawing direction but reduces the elongation at break, whereas an increase in elongation at break is often noticed in oriented amorphous polymer fibers ${ }^{18}$. As an example, hot drawn poly(L-lactide-co-glycolide) (PLGA) 80/20 monofilament fibers with a diameter of $0.4 \mathrm{~mm}$ had a tensile strength of $377 \mathrm{MPa}^{19}$. On the other hand, the relative decrease in inherent viscosity of our extruded poly(DTE carbonate) fiber $(0.58 \pm 0.03 \mathrm{dl} / \mathrm{g})$ compared to the raw material $(0.89 \pm 0.01 \mathrm{dl} / \mathrm{g})$ was $35 \%$, indicating that some polymer degradation has occurred during extrusion. The result is in line with a comprehensive study of oriented PLGA 85/15 fibers reporting relative post-extrusion i.v. value decreases between $29 \%$ and $56 \%$ depending on the processing conditions and raw material batch ${ }^{20}$.

The water uptake properties of PCL (Figure 3) were tested to estimate the water barrier properties of the extrusion coating. An equilibrium water uptake of PCL was noticed to be around 0.30-0.35 wt- $\%$, which is low compared to many of the other biodegradable polymers whose typical water uptake is in the order of $1 \mathrm{wt}-\%^{21}$. Correspondingly, PCL water uptake after 60 days has been reported to be $0.5 \%$, whereas the respective value for other tested polymers like poly(butylene succinate) (PBS) and poly(lactic acid) (PLA) were approximately $1.5 \%{ }^{22}$. Due to these properties, PCL has been previously described as a good material candidate for encapsulating sensors ${ }^{23}$. 


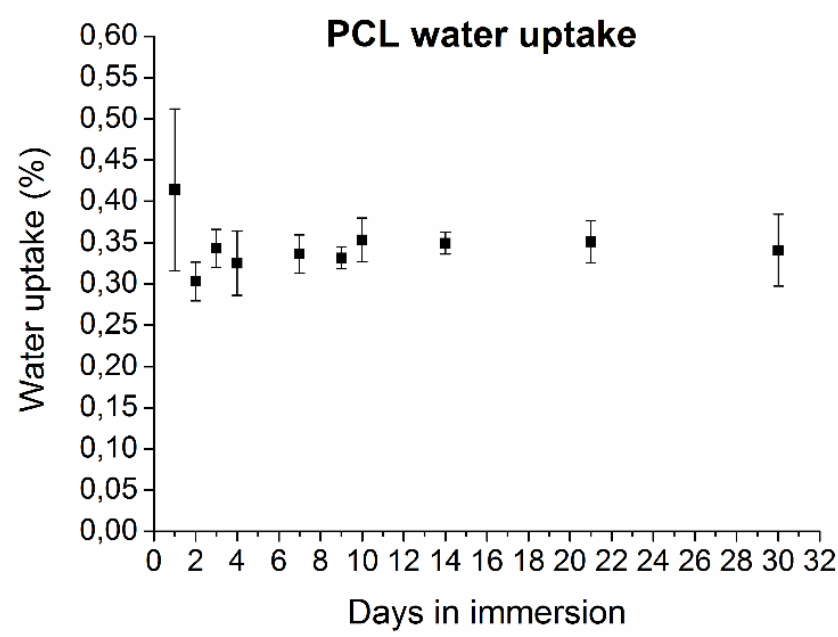

Figure 3. Water uptake behavior of poly(caprolactone) sheets $(n=5)$ immersed in Sörensen buffer solution. The error bars denote standard deviations.

\section{Resistance measurements}

The average electrical resistance of the wires after e-beam evaporation of $\mathrm{Mg}$ was $0.9 \pm 0.1 \Omega \mathrm{cm}^{-1}$, as shown in Figure 4a. A slight increase in resistance to $1.1 \pm 0.2 \Omega \mathrm{cm}^{-1}$ was noticed after extruding the PCL sheath onto the wire. This might be due to the detachment of Mg from the core fiber in some locations as seen in the SEM image (Figure 2). All of the tested wires $(\mathrm{n}=21)$, however, remained electrically conductive after the extrusion coating process, addressing the applicability of the fabrication method.

The resistance values of our conductive wires can be considered reasonable, factoring in the electrical properties of magnesium and its dimensions in our application. With thinner Mg layers, the resistance increases, which might be problematic in some applications. For example, a $0.5 \mu \mathrm{m}$ thick Mg layer on a poly(DTE carbonate fiber) resulted in a resistance of about $10 \Omega \mathrm{cm}^{-1}$ (Table S2), which would impair their application as RF coils. On the other hand, a $0.5 \mu \mathrm{m}$ thick $\mathrm{Cu}$ layer on a fiber provided a much lower resistance of approximately $0.5 \Omega \mathrm{cm}^{-1}$. In the literature, the 
resistance of a copper wire based non-resorbable implantable wire of approximately $0.1 \Omega \mathrm{cm}^{-1}$ has been reported ${ }^{1}$. On the other end of the scale, conductive polymer based yarns ${ }^{24}$ and wires ${ }^{25}$ have been reported with resistances of $77 \Omega \mathrm{cm}^{-1}$ to around $2550 \Omega \mathrm{cm}^{-1}$, respectively.

Coiling wires around a glass rod $(\mathrm{r}=3.5 \mathrm{~mm})$ did not significantly increase the resistance of the wires and none of the tested wires $(\mathrm{n}=5)$ were destroyed during the experiment. Thus, the bending radius that the wires withstand without breaking the magnesium layer is $3.5 \mathrm{~mm}$ or less, illustrating the flexibility of the prototype wire.

(a) Effect of extrusion coating $(\mathrm{n}=\mathbf{2 1})$

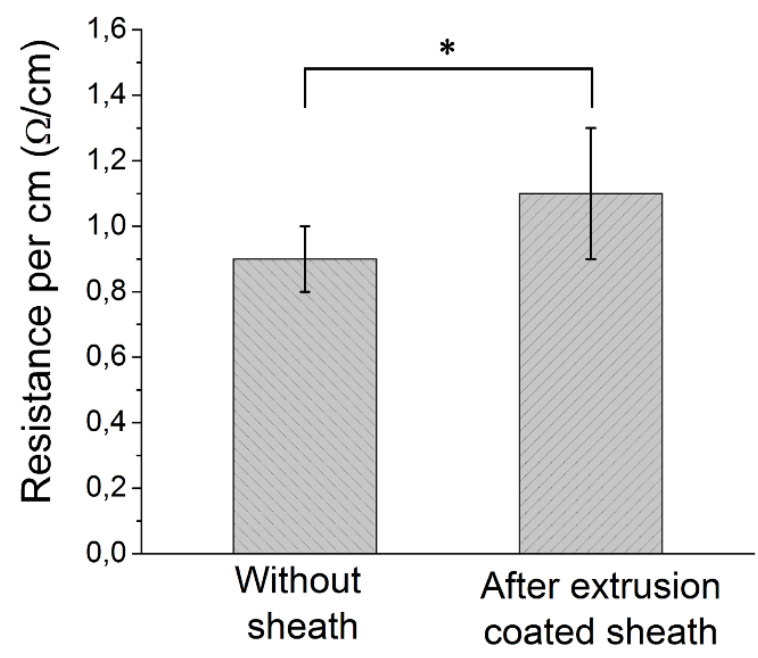

(b) Effect of bending ( $n=5)$

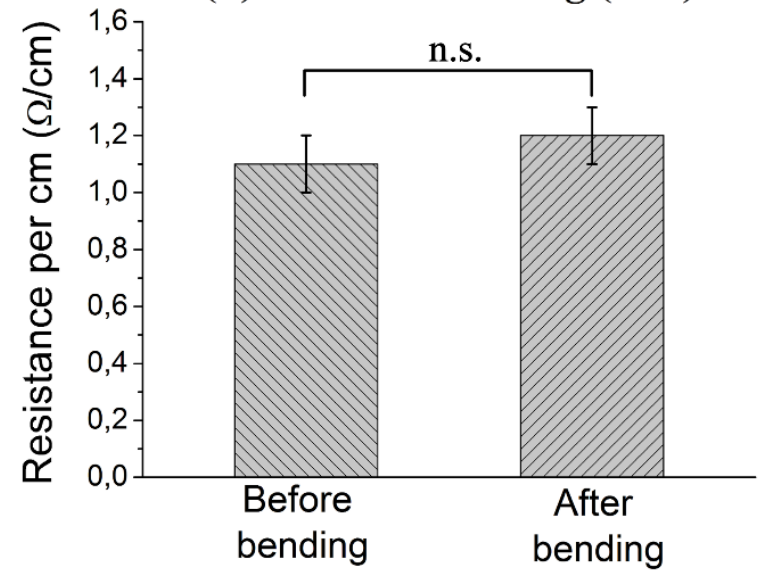


Figure 4. The average resistances of conductive wires (a) before and after extrusion coating (b) before and after bending (bending radius $\mathrm{r}=3.5 \mathrm{~mm}$ ). The error bars denote standard deviations. A paired Student's t-test was used to assess the statistical significance: ${ }^{*} \mathrm{p}<0.05$, n.s. $=$ not significant.

Immersing wires in Sörensen buffer solution was done to evaluate the water barrier properties of the PCL coating and to approximate the functional lifetime of the wires in vitro. The silver epoxy adhesive connection at the tip of the wires was used to protect the thin $\mathrm{Mg}$ layer during repeated measurements. However, this connection led to a slight resistance increase in the other samples and a larger effect in sample \#4. After the first 24 hours of immersion in buffer solution, all the samples showed increased resistances (Figure 5). This is probably due to the low amount of absorbed water $(\sim 0.35$ wt- $\%)$ inside the PCL coating. The absorbed water might initiate the corrosion of $\mathrm{Mg}$ or facilitate the delamination of the PCL coating ${ }^{21}$, thus leading to some $\mathrm{Mg}$ detachment. For comparison, wires without PCL coating lose their conductivity completely in a few minutes in buffer solution (data not shown).

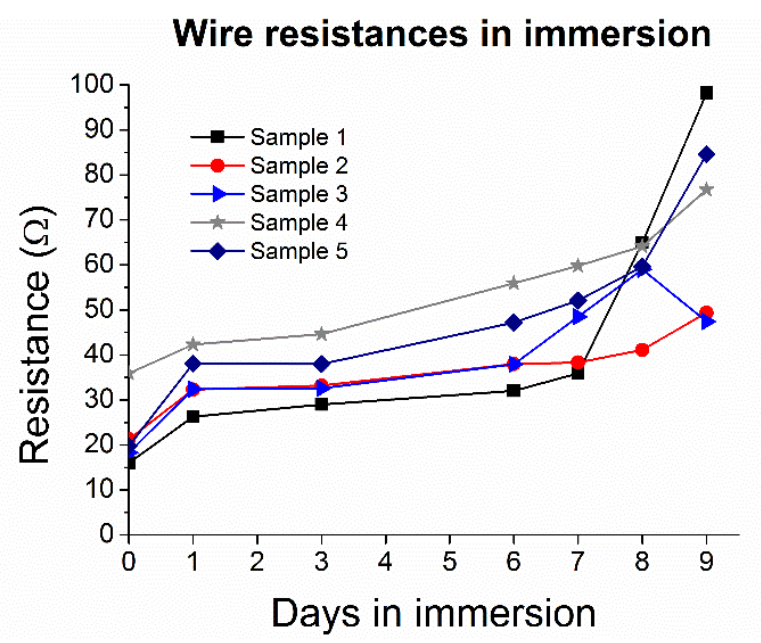

Figure 5. The resistances of PCL-coated conductive wires immersed in Sörensen buffer solution at $+37^{\circ} \mathrm{C}$. 
The samples demonstrate a moderate resistance increase between the first and the sixth day. After one week the measurement results start to drift, probably due to the noticed flaking of the silver epoxy adhesive layer. The observed flaking of the Ag epoxy was probably caused by repeated mechanical stress that was derived from the hook clip probes. All the wires were still conductive $(<100 \Omega)$ after 9 days. At the 10-day time-point, the first two wires have lost their conductivity (not shown). Three days later, the three samples were still conductive. After 14 days, the hydrolysis was terminated and the conductivities were measured directly from the $\mathrm{Mg}$ layer. Only samples $\# 3$ and \#4 had remained conductive with the resistances of $120 \Omega\left(10.7 \Omega \mathrm{cm}^{-1}\right)$ and $175 \Omega(14.1$ $\left.\Omega \mathrm{cm}^{-1}\right)$, respectively.

The results indicate that water does not penetrate the PCL coating excessively during the first week of immersion. Furthermore, by choosing a more corrosion resistant metal such as iron or zinc, the functional lifetime of the wire could probably be significantly extended. Other tuning possibilities include e-beam evaporating both sides of the core fiber leading to a conductive cable with two separate conductors in one fiber, or using sputter deposition to cover the whole fiber with metal.

\section{Planar coil RF measurements}

To demonstrate the usability of the developed fabrication methods, bioresorbable coils were built and their self-resonance frequencies were inductively measured. Both magnesium and copper based coils were clearly readable through the Petri dish, but the copper wire based reference coils produced higher and sharper resonance peaks compared to the bioresorbable coils (Figure 6a). This was an expected result considering the thickness and conductivity of the magnesium layer in comparison with the commercial copper wire with a conductor diameter of $0.40 \mathrm{~mm}$. 
Correspondingly, one of the key challenges in producing high quality factor biodegradable RF resonators is the conductivity of the materials ${ }^{26}$.

The varying resonance frequencies of the coils were probably caused by the manual assembly; the turns are difficult to position identically, which leads to different capacitances between the turns. The changes in capacitance lead to significant changes in the resonance frequency.

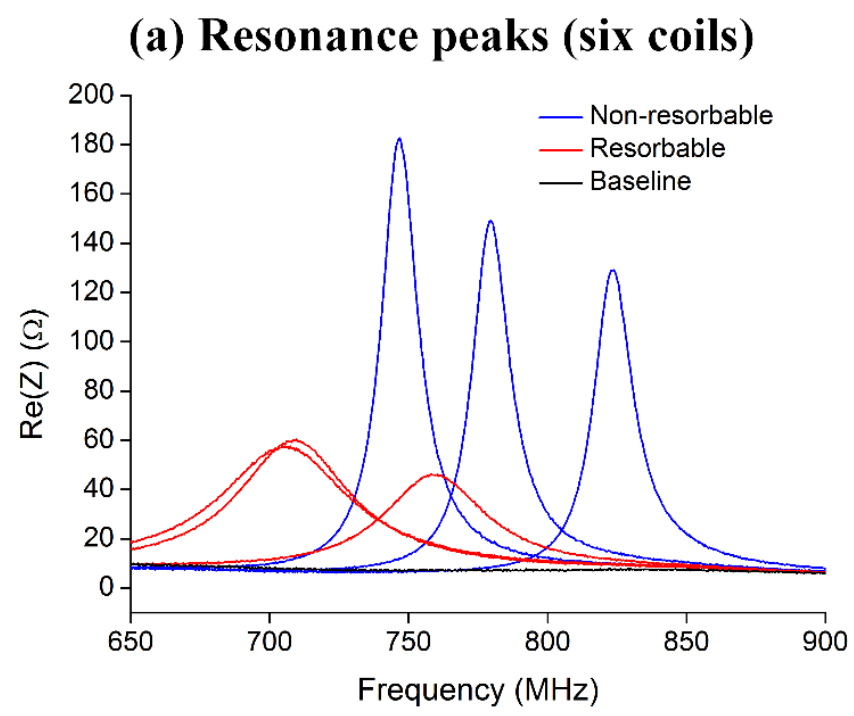

(b) The effect of reading distance

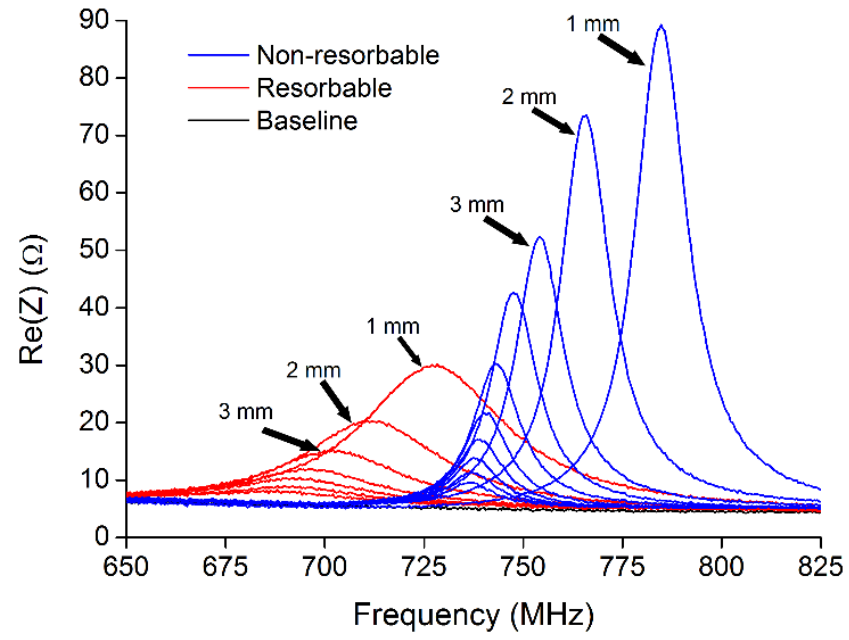


Figure 6. The resonance peaks of (a) all the fabricated planar coils, including three resorbable coils and three nondegradable copper wire based reference coils (b) one resorbable and one non-resorbable coil measured with increasing reading distance through glass microscopy slides.

The reading distance of the bioresorbable coil was approximately 4-5 $\mathrm{mm}$ (Figure 6b), after which it became challenging to distinguish the resonance curve from the background noise. The higher conductivity of the non-degradable coils lead to an approximately twice as high reading distance compared with the $\mathrm{Mg}$ based bioresorbable coils. The resonance frequencies increase with decreasing reading distance due to stronger inductive coupling, which is in agreement with previous simulation results ${ }^{27}$.

In order to use these kind of structures in practical sensor applications, the manual assembly of the coils should be replaced with a more consistent process, for example by using more sophisticated templates. This would minimize the differences in the geometry of the RF coils. In typical applications the measured quantities are linked to the change of the resonance frequency. In our measurements, the reading distance affected the detected resonance frequency. This phenomenon was caused by the fact that in this setup the self-resonance frequency of the reader coil (not shown) was lower than the measured frequencies. Thus, the readout configuration should be optimized and in addition, the errors could be mitigated by adapting a method for reading distance compensation $^{28}$. In the future, physical changes in the coil dimensions and their effect to the resonance frequency could be utilized in a wireless sensor for example to monitor the dimensions of stents or tubular organs.

\section{CONCLUSIONS}


The design and processing strategy of a bioresorbable implantable wire with minimal metal content has been described. This approach might be beneficial in signal and energy transfer applications where higher metal content would be problematic considering their toxicity, degradation rate or the degradation by-products. In addition, the manually fabricated test coils were wirelessly readable and their improved versions could be used in the future as biodegradable sensors. The lifetime of the wires immersed in buffer solution was more than one week, although only a few micrometers thick layer of extremely fast corroding magnesium was used. To conclude, the presented bioresorbable prototype wires showed promising properties for an application as implantable transient wires.

\section{ASSOCIATED CONTENT}

Supporting Information. Dimensions of the extruder die, the composition and instructions for preparing the Sörensen buffer solution, resistances for poly(DTE carbonate) fibers with different metal layers.

\section{AUTHOR INFORMATION}

\section{Corresponding Author}

* Correspondence should be addressed to Aleksi Palmroth; aleksi.hanninen@tut.fi

ORCID: Aleksi Palmroth 0000-0002-8574-6922

\section{Funding Sources}

This work was funded by Business Finland as a part of the Human Spare Parts research program and by the Finnish Cultural Foundation's Kalle and Dagmar Välimaa fund.

\section{Notes}


The authors declare no competing financial interest.

\section{ACKNOWLEDGMENT}

We would like to thank Mr. Heikki Liejumäki for performing the inherent viscosity analysis and Mrs. Suvi Heinämäki for technical assistance in the laboratory, as well as Ms. Laura Johansson for her help with the extrusion parameters. 


\section{REFERENCES}

(1) Kim, S.-H.; Moon, J.-H.; Kim, J.; H., Jeong; S. M.; Lee, S.-H. Flexible, Stretchable and Implantable PDMS Encapsulated Cable for Implantable Medical Device. Biomed. Eng. Lett. 2001, 1, 199-203. DOI: 10.1007/s13534-011-0033-8.

(2) Ong, K. G.; Grimes, C. A.; Robbins, C. L.; Singh, R. S. Design and application of a wireless, passive, resonant-circuit environmental monitoring sensor. Sensors Actuators A Phys. 2001, 93, 33-43. DOI: 10.1016/S0924-4247(01)00624-0.

(3) Kim, S.; Yoo, S. Comparison of planar type coils for efficient power supply to implantable devices. Biomed. Eng. Lett. 2012, 2, 179-185. DOI: 10.1007/s13534-012-0070-y.

(4) Kim, D.-H.; Kim, Y.-S.; Amsden, J.; Panilaitis, B.; Kaplan, D. L.; Omenetto, F. G.; Zakin, M. R.; Rogers, J. A. Silicon electronics on silk as a path to bioresorbable, implantable devices. Appl. Phys. Lett. 2009, 95, 133701. DOI: 10.1063/1.3238552.

(5) Nakiri, T.; Kawachi, Y.; Honda, M.; Imoto, K.; Yamakita, T.; Tajitsu, Y. Development of Electric Wire Using Biodegradable Polymer. IEEE Trans. Ind. Appl. 2007, 43, 1069-1074. DOI: 10.1109/TIA.2007.900455.

(6) Miyauchi, M.; Miao, J.; Simmons, T. J.; Lee J.-W.; Doherty, T. V.; Dordick, J. S.; Linhardt, R. J. Conductive cable fibers with insulating surface prepared by co-axial electrospinning of multi-walled nanotubes and cellulose. Biomacromolecules 2010, 11, 2440-2445. DOI: $10.1021 / \mathrm{bm} 1006129$.

(7) Kang, S.-K.; Murphy, R. K. J.; Hwang, S.-W.; Lee, S. M.; Harburg, D. V.; Krueger, N. A.; Shin, J.; Gamble, P.; Cheng, H.; Yu, S.; Liu, Z.; McCall, J. G.; Stephen, M.; Ying, H.; Kim, 
J.; Park, G.; Webb, R. C.; Lee, C. H.; Chung, S.; Wie, D. S.; Gujar, A. D.; Vemulapalli, B.; Kim, A. H.; Lee, K.-M.; Cheng, J.; Huang, Y.; Lee, S. H.; Braun, P. V.; Ray, W. Z.; Rogers, J. A. Bioresorbable silicon electronic sensors for the brain. Nature 2016, 530, 71-76. DOI: 10.1038/nature16492.

(8) Curry, E. J.; Ke, K.; Chorsi, M. T.; Wrobel, K. S.; Miller III, A. N.; Patel, A.; Kim, I.; Feng, J.; Yue, L.; Wu, Q.; Kuo, C.-L.; Lo, K. W.-H.; Laurencin, C. T.; Ilies, H.; Purohit, P. K.; Nguen, T. D. Biodegradable Piezoelectric Force Sensor. Proc. Natl. Acad. Sci. 2018, 115, 909-914. DOI: 10.1073/pnas.1710874115.

(9) Staiger, M. P.; Pietak, A. M.; Huadmai, J.; Dias, G. Magnesium and its alloys as orthopedic biomaterials: A review. Biomaterials 2006, 27, 1728-1734. DOI: $10.1016 /$ j.biomaterials.2005.10.003.

(10) Asgari, M.; Hang, R.; Wang, C.; Yu, Z.; Li, Z.; Xiao, Y. Biodegradable Metallic Wires in Dental and Orthopedic Applications: A Review. Metals 2018, 8. DOI: $10.3390 /$ met8040212.

(11) Katarivas Levy, G.; Goldman, J.; Aghion, E. The Prospects of Zinc as a Structural Material for Biodegradable Implants-A Review Paper. Metals 2017, 7, 1-18. DOI: $10.3390 / \operatorname{met} 7100402$.

(12) Lam, C. X. F.; Hutmacher, D. W.; Schantz, J.-T.; Woodruff, M. A.; Teoh, S. H. Evaluation of polycaprolactone scaffold degradation for 6 months in vitro and in vivo. J. Biomed. Mater. Res. Part A 2008, 90A, 906-919. DOI: 10.1002/jbm.a.32052.

(13) Samitsu, S.; Zhang, R.; Peng, X.; Krishnan, M. R.; Fujii, Y.; Ichinose, I. Flash freezing 
route to mesoporous polymer nanofibre networks. Nat. Commun. 2013, 4, 2653. DOI: 10.1038/ncomms3653.

(14) Yousaf, A.; Khan, F. A.; Reindl, L. M. Passive Wireless Sensing of Micro coil parameters in fluidic environments. Sensors Actuators, A Phys. 2012, 186, 69-79. DOI: 10.1016/j.sna.2012.01.032.

(15) Bourke, S. L.; Kohn, J.; Dunn, M. G. Preliminary Development of a Novel Resorbable Synthetic Polymer Fiber Scaffold for Anterior Cruciate Ligament Reconstruction. Tissue Eng. 2004, 10, 43-52. DOI: 10.1089/107632704322791682.

(16) Park, Y.-S.; Choi, K.-H; Kim, H.-K. Room temperature flexible and transparent ITO/Ag/ITO electrode grown on flexile PES substrate by continuous roll-to-roll sputtering for flexible organic photovoltaics. J. Phys. D. Appl. Phys. 2009, 42, 235109. DOI: $10.1088 / 0022-3727 / 42 / 23 / 235109$.

(17) Asikainen, A. J.; Pelto, M.; Noponen, J.; Kellomäki, M.; Pihlajamäki, H.; Lindqvist, C.; Suuronen, R. In vivo degradation of poly(DTE carbonate) membranes. Analysis of the tissue reactions and mechanical properties. J. Mater. Sci. Mater. Med. 2008, 19, 53-58. DOI: $10.1007 / \mathrm{s} 10856-007-3134-2$.

(18) La Mantia, F. P., Ceraulo, M., Mistretta, M. C. \& Morreale, M. Effect of Cold Drawing on Mechanical Properties of Biodegradable Fibers. J. Appl. Biomater. Funct. Mater. 15, 7076 (2017). DOI: 10.5301/jabfm.5000328.

(19) Juuti, H.; Kotsar, A.; Mikkonen, J.; Isotalo, T.; Talja, M.; Tammela, T. L.; Törmälä, P.; Kellomäki, M. The Effect of pH on the Degradation of Biodegradable Poly(L-Lactide-Co- 
Glycolide) 80/20 Urethral Stent Material In Vitro. J. Endourol. 2011, 26, 701-705. DOI: 10.1089/end.2011.0199.

(20) Paakinaho, K.; Heino, H.; Väisänen, J.; Törmälä, P; Kellomäki, M. Effects of lactide monomer on the hydrolytic degradation of poly(lactide-co-glycolide) $85 \mathrm{~L} / 15 \mathrm{G}$. J. Mech. Behav. Biomed. Mater. 2011, 4, 1283-1290. DOI: 10.1016/j.jmbbm.2011.04.015.

(21) Lyu, S.; Untereker, D. Degradability of polymers for implantable biomedical devices. Int. J. Mol. Sci. 2009, 10, 4033-4065. DOI: 10.3390/ijms10094033.

(22) Correlo, V. M.; Pinho, E. D.; Pashkuleva, I.; Bhattacharya, M.; Neves, N.M.; Reis, R. L. Water Absorption and Degradation Characteristics of Chitosan-Based Polyesters and Hydroxyapatite Composites. Macromol. Biosci. 2007, 7, 354-363. DOI: 10.1002/mabi.200600233.

(23) Salpavaara, T.; Lekkala, J.; Khan, S.; Ellä, V.; Kellomäki, M. Biodegradable encapsulation for inductively measured resonance circuit. In 2012 IEEE 12th International Conference on Bioinformatics \& Bioengineering (BIBE) 323-327. DOI: 10.1109/BIBE.2012.6399644.

(24) Amba Sankar, K.N.; Mohanta, K. Dwindling the resistance value of PEDOT:PSS - coated on fabric yarns. AIP Conf. Proc., 2016, 1731, 120019. DOI: 10.1063/1.4948091.

(25) Nagamine, K.; Sato, H.; Kai, H.; Kaji, H.; Kanzaki, M.; Nishizawa, M. Contractile Skeletal Muscle Cells Cultured with a Conducting Soft Wire for Effective, Selective Stimulation. Sci. Rep. 2018, 8, 2253. DOI: 10.1038/s41598-018-20729-y.

(26) Boutry, C. M.; Chandrahalim, H.; Streit, P.; Schinhammer, M.; Hänzi, A. C.; Hierold, C. Towards biodegradable wireless implants. Philos. Trans. R. Soc. A Math. Phys. Eng. Sci. 
2012, 370, 2418 LP-2432. DOI: 10.1098/rsta.2011.0439.

(27) Salpavaara, T.; Lekkala, J. A Model Based Analysis of the Measurement Errors in Inductively Coupled Passive Resonance Sensors. In XXI IMEKO World Congress 'Measurement in Research and Industry' 2015.

(28) Salpavaara, T.; Verho, J.; Kumpulainen, P; Lekkala, J. Readout methods for an inductively coupled resonance sensor used in pressure garment application. Sensors Actuators, A Phys. 2011, 172, 109-116. DOI: 10.1016/j.sna.2011.02.051. 


\section{For Table of Contents Use Only}

Title: Bioresorbable conductive wire with minimal metal content

Authors: Aleksi Palmroth, Timo Salpavaara, Inari Lyyra, Mart Kroon, Jukka Lekkala, Minna Kellomäki

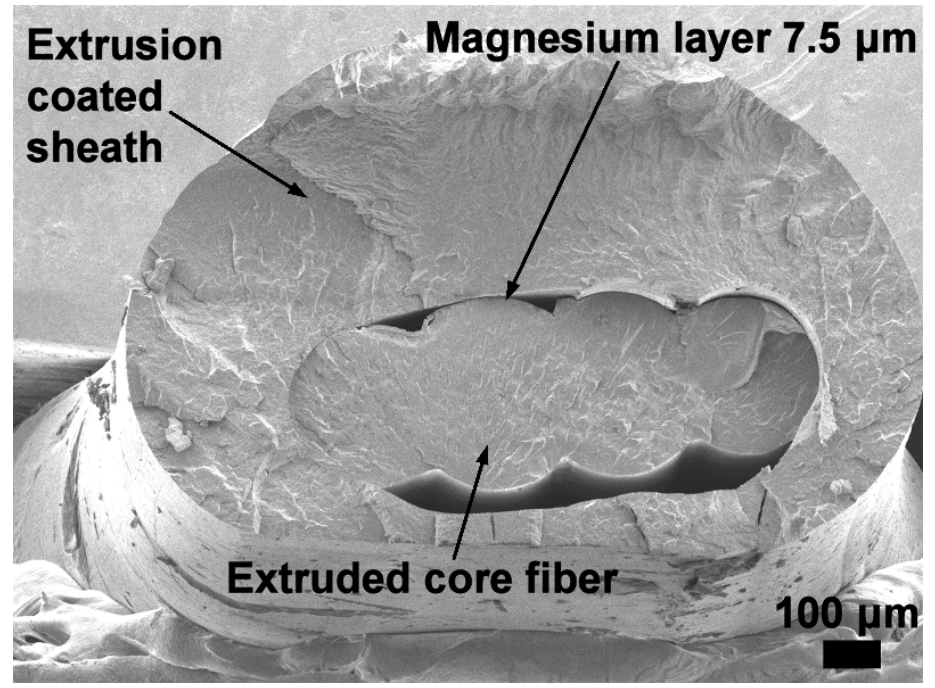

